\title{
Sex Steroids and Bone Mass in Older Men \\ Positive Associations with Serum Estrogens and Negative Associations with Androgens
}

\author{
Charles W. Slemenda, ${ }^{\star}$ Christopher Longcope, ${ }^{\ddagger}$ Lifen Zhou, ${ }^{\star}$ Siu L. Hui, ${ }^{\star}$ Munro Peacock, ${ }^{\star}$ and C. Conrad Johnston* \\ $*$ Department of Medicine, Indiana University School of Medicine, Indianapolis, Indiana 46202; and ${ }^{\ddagger}$ Department of Medicine and \\ Department of Obstetrics and Gynecology, University of Massachusetts, Worcester, Massachusetts 01655
}

\begin{abstract}
The purpose of this study was to determine whether bone density in older men was associated with serum sex steroids or sex hormone binding globulin (SHBG). Bone density and sex steroids were measured in men over age 65 at 6-mo intervals for an average of $2.1 \mathrm{yr}$. Bone density was significantly positively associated with greater serum E2 concentrations $(+0.21<r<+0.35 ; 0.01<P<0.05)$ at all skeletal sites. There were weak negative correlations between serum testosterone and bone density $(-0.20<r<-0.28 ; 0.03<P<$ $0.10)$ at the spine and hip. SHBG was negatively associated only with bone density in the greater trochanter $(r=-0.26$, $P<0.05$ ). Greater body weight was associated with lower serum testosterone and SHBG, and greater E2. Because of these associations, regression models which adjusted for age, body weight, and serum sex steroids were constructed; these accounted for 10-30\% of the variability in bone density, and showed consistent, significant positive associations between bone density and serum E2 concentrations in men, even after adjustments for weight and SHBG. These data suggest that estrogens may play an important role in the development or maintenance of the male skeleton, much as is the case for the female skeleton. These data also indicate that, within the normal range, lower serum testosterone concentrations are not associated with low bone density in men. (J. Clin. Invest. 1997. 100:1755-1759.) Key words: bone density • estrogens • testosterone $\cdot$ sex hormone binding globulin $\bullet$ men
\end{abstract}

\section{Introduction}

Osteoporosis is common in older men. Although often not recognized because of their shorter life spans and greater peak bone mass, men have about half the age-specific risk of experiencing osteoporotic fractures as do women (1-5). There is some evidence that this gap may be closing, as fracture rates in men in some areas of the United States and Europe continue to increase while those for women appear to have stabilized (2, 3 ). Because the focus of osteoporosis research has been on

Address correspondence to C. Conrad Johnston, Jr., M.D., Indiana University School of Medicine, 545 Barnhill Drive, Emerson Hall 421, Indianapolis, IN 46202-5124. Phone: 317-274-8554; FAX: 317-274-4311.

Received for publication 27 January 1997 and accepted in revised form 14 August 1997.

J. Clin. Invest.

(C) The American Society for Clinical Investigation, Inc. 0021-9738/97/10/1755/05 \$2.00

Volume 100, Number 7, October 1997, 1755-1759

http://www.jci.org women, much less is known regarding those factors that may influence bone mass or loss in older men (1). Despite the relative lack of large prospective studies of osteoporotic fractures in men, low bone mass (4), risk factors for falls (6), and other factors believed to be associated with fractures in women appear to be similarly associated in men.

Many factors which influence bone mass in men appear to be similar to those which determine bone mass in women. Genetic influences on bone mass appear to be similar in men and women $(7,8)$. As with women, physical activity and greater strength are also associated with greater bone mass in men (9). Recent prospective studies have identified cigarette smoking and immoderate alcohol consumption as factors which contribute to bone loss from the radius in older men (10), but factors influencing bone loss at other sites have not been well studied, primarily because bone loss in men proceeds more slowly than in women, and the precision of methods for assessing these slower rates of loss requires long periods of follow-up to obtain stable estimates of rates of loss.

The importance of sex steroids in the development and maintenance of the skeleton in women is unquestioned. In particular, estrogen deficiency at any age is associated with negative skeletal effects, with only rare exceptions. Studies of sex steroids and gonadal function in men have been much less common, and have focused primarily on androgens. Although weak correlations between androgens and bone density have been reported (11), others have failed to confirm this observation $(12,13)$. Similarly, there have been conflicting reports regarding the value of testosterone treatment of men without clinically evident hypogonadism. A nonrandomized study of testosterone injections in eugonadal men with osteoporotic fractures found improvements in spine but not hip density (14), whereas a clinical trial of transdermal testosterone failed to find improvements (15). Estrogens in men have rarely been studied with respect to bone density. Recently it was reported that a young adult male with a genetically defective estrogen receptor had severe osteopenia despite continuing longitudinal growth to a height of greater than $2 \mathrm{~m}$ (16), reflecting on several previously unrecognized aspects of the importance of estrogen in the male skeleton. The data from this case report suggest that estrogens play a role in the closure of the epiphyses in male as well as female adolescents, and that the development of normal peak bone mass in men may also depend in part on estrogens. The study described below was designed to address questions regarding the importance of both estrogens and androgens in bone density in healthy older men.

\section{Methods}

Overview. Healthy older, nonsmoking men were studied prospectively over a period of $1-4 \mathrm{yr}$ to determine the associations among sex steroids, and between sex steroids and bone density.

Subjects. 93 healthy men over age 55 were recruited from several sources, including the spouses of women enrolled in our prospective 
studies of bone mass and sex steroids (17), and respondents to newspaper advertisements and other public notices. All participants provided informed consent, and all procedures were approved by the Indiana University Institutional Review Board. Men were excluded if they had any medical conditions which might affect bone density (e.g., hypogonadism, thyroid disease) or were taking any medications known to affect the skeleton (including corticosteroids, thyroid hormones, estrogens or androgens, treatments for prostatic disease; diuretics were permitted). Six were smokers. They had lower bone density than the nonsmokers, but due to the small sample these men are excluded from further analyses and discussion.

Bone mass measurements. All measurements were made at $\sim 6$-mo intervals; there was an average of 5.5 measurements per subject over slightly more than $2 \mathrm{yr}$. The midradius bone mineral density (BMD) ${ }^{1}$ was measured by single photon absorptiometry (SPA) (Lunar Corp., Madison, WI), and the lumbar spine (L2-L4) and hip BMDs (femoral neck, trochanter, and Ward's triangle) by dual energy $\mathrm{x}$-ray absorptiometry (DXA) (Lunar Corp.). Short-term in vivo reproducibility has been $1-2 \%$ in adults depending on site (1.04\% L2-L4 to 1.8\% Ward's triangle), and long-term reproducibility (i.e., the coefficient of variation about the slope of repeated measurements within subjects over $3 \mathrm{yr}$ ) between $1.4 \%$ (radius) and $4 \%$ (Ward's triangle).

Each subject's mean BMD over an average of $2.1 \mathrm{yr}$ was calculated. Rates of change in BMD were estimated by least squares regression through each subject's BMD measurements of this same period of follow-up. Only those subjects with three or more measurements were included in the calculation of rates of bone loss. There was significant bone loss from the radius and hip, but not from the spine. However, rates of bone loss were relatively variable over this short period of time. Therefore, for analyses of bone loss subjects were classified into two groups: those who lost at least $1 \%$ per year, and the remainder.

Sex steroids measurements. Blood was drawn at each visit (without respect to time of day). Measurements were made of estrone (E1), estradiol (E2), estrone sulfate $\left.\left(\mathrm{E}_{\mathrm{SO}}\right)_{4}\right)$, testosterone $(\mathrm{T})$, androstenedione (A), free E2 and T, sex hormone binding globulin (SHBG), dehydroepiandrosterone (DHEA) and dehydroepiandrosterone sulfate (DHEAS), and luteinizing and follicle stimulating hormones (17). The percent free $\mathrm{T}$ and free $\mathrm{E} 2$ were directly measured by centrifugal ultracentrifugation using a modification of the method of Hammond and colleagues as we have described previously (18). The total free $\mathrm{T}$ was then determined by multiplying the measured percent free $\mathrm{T}$ times the total $\mathrm{T}$ divided by 100 . For each subject the mean value of each sex steroid was calculated but, unlike bone mass, rates of change in the sex steroids were not considered. There was an average of 5.1 samples per subject over $2 \mathrm{yr}$ or more. Over the relatively short period of study the biological variability within subjects was too great to obtain stable estimates of rates of change in the sex steroids.

Bone turnover. Measurements of urinary markers of bone turnover were made at each visit. Urinary collagen cross-links (NTX, Ostex International, Inc.) were measured by ELISA on nonfasting, spot samples using a commercially available kit (19).

Analyses. Variables were examined for distributional characteristics. Most sex steroids were transformed (log, base e) to produce more normal distributions, and correlation and regression analyses were performed on the transformed data. Univariate associations between sex steroids (log-transformed) and BMD were examined with simple correlations. Partial correlations and regression models were used to examine the joint effects of multiple predictors on BMD.

1. Abbreviations used in this paper: $\mathrm{A}$, androstenedione; $\mathrm{BMD}$, bone mineral density; DHEA, dehydroepiandrosterone; DHEAS, DHEA sulfate; E1, estrone; $\mathrm{E}_{\mathrm{SO}}$, estrone sulfate; E2, estradiol; SHBG, sex hormone binding globulin; T, testosterone.

\section{Results}

Descriptive data are provided in Table I. Serum estrogen concentrations in these men were similar to women in this same age range (17), whereas androgen concentrations were 30-40 times higher in the men.

The correlations among the sex steroids are shown in Table II, as well as their correlations with weight and age. Serum concentrations of the estrogens (E1 and E2) were strongly positively correlated with each other $(r=0.60, P<0.001)$, and E1 was also positively correlated with the androstenedione (reflecting the aromatase-mediated conversion of A to E1), DHEA, and DHEAS $(0.33<r<0.59$, all $P<0.01)$. E2 was only weakly correlated with other sex steroids. Serum $\mathrm{T}$ had a strong positive association with SHBG $(r=0.65, P<0.001)$, and a negative association with body weight $(r=-0.42, P<$ 0.001 ). In regression models containing weight and serum $T$ concentrations, only serum $\mathrm{T}$ was a significant predictor of SHBG, accounting for $40 \%$ of the variability in its serum concentrations (regression models not shown).

The correlations between mean serum sex steroids and mean BMD and body size over the period of study are shown in Table III. BMD was positively associated with weight, significantly so at the spine and proximal femur sites. Serum E2 concentrations were significantly positively correlated with bone density at all sites $(0.21<r<0.33)$. This was the only sex steroid significantly positively correlated with bone density. There were negative associations between BMD and T, A, DHEA, and SHBG of varying strengths and statistical significance (Table III), but generally these associations were weaker than the positive estrogen associations. Although there were no correlations between measures of bone size or projected bone area and serum estrogen concentrations, serum testosterone concentrations were negatively correlated with bone size measurements at all skeletal sites. These correlations ranged from $r=$ -0.18 (radius width, $P=0.08$ ) to $r=-0.28$ (trochanteric area, $P<0.01)$.

To examine the independence of these serum sex steroids and body size as predictors of BMD, two approaches were taken. First, subjects were stratified into those above and be-

\section{Table I. Descriptive Data, Means $\pm S D$}

$\begin{array}{lc}\text { Age, } \mathrm{yr} & 67.3 \pm 4.3 \\ \text { Height, } \mathrm{cm} & 175.4 \pm 6.1 \\ \text { Weight, } \mathrm{kg} & 84.9 \pm 13.7 \\ \mathrm{E} 1, \mathrm{pg} / \mathrm{ml} & 36.0 \pm 13.4 \\ \mathrm{E} 2, \mathrm{pg} / \mathrm{ml} & 35.4 \pm 11.8 \\ \mathrm{E} 1 \mathrm{SO}, \mathrm{pg} / \mathrm{ml} & 292 \pm 135 \\ \mathrm{~T}, \mathrm{ng} / \mathrm{ml} & 4.2 \pm 1.6 \\ \mathrm{~A}, \mathrm{ng} / \mathrm{ml} & 1.12 \pm 0.43 \\ \mathrm{DHEA}, \mathrm{ng} / \mathrm{ml} & 0.98 \pm 0.66 \\ \mathrm{DHEAS}, \mu \mathrm{g} / \mathrm{ml} & 1.24 \pm 0.76 \\ \mathrm{SHBG}, \mathrm{nM} / \mathrm{liter} & 43.4 \pm 21.1 \\ & \\ \text { Midradius, g/cm } & \\ \text { L2-L4, g/cm } & 0.78 \pm 0.07 \\ \text { Femoral neck, g/cm } & 1.31 \pm 0.20 \\ \text { Trochanter, } \mathrm{g} / \mathrm{cm}^{2} & 0.94 \pm 0.14 \\ & 0.90 \pm 0.14\end{array}$

$n=87$. 
Table II. Correlations: Sex Steroids and Body Size

\begin{tabular}{lccccccc}
\hline & \multicolumn{7}{c}{ Sex steroids } \\
\cline { 2 - 8 } & E1 & E2 & T & A & DHEA & DHEAS & SHBG \\
\hline Height & 0.08 & -0.08 & $-0.29^{*}$ & 0.02 & 0.02 & $0.18^{\ddagger}$ & $-0.22^{\S}$ \\
Weight & 0.05 & 0.15 & $-0.42^{*}$ & -0.08 & -0.15 & 0.05 & $-0.29^{*}$ \\
E1 & - & $0.60^{*}$ & 0.01 & $0.47^{*}$ & $0.33^{*}$ & $0.59^{*}$ & -0.09 \\
E2 & & - & 0.13 & $0.24^{\S}$ & 0.09 & $0.24^{\S}$ & 0.02 \\
T & & & - & $0.30^{\|}$ & $0.35^{\|}$ & 0.10 & $0.65^{*}$ \\
A & & & & - & $0.66^{*}$ & $0.45^{*}$ & 0.05 \\
DHEA & & & & - & $0.56^{*}$ & $0.27^{\|}$ \\
DHEAS & & & & & - & 0.01 \\
& & & & & & & \\
\hline
\end{tabular}

${ }^{\ddagger} 0.05<P<0.10 ;{ }^{\S} P<0.05 ;{ }^{\|} P<0.01 ; * P<0.001$.

low the median weight, and then into those above and below the median serum E2 concentration. The results of this analysis for the femoral neck are shown in Fig. 1. Those men above the median for both weight and E2 $(n=24)$ had BMDs 0.14 $\mathrm{g} / \mathrm{cm}^{2}$ (1 SD) higher than those below the median for both of these variables $(n=23)$. Similar patterns were seen for all skeletal sites (L2-L4 BMD difference $=0.11 \mathrm{~g} / \mathrm{cm}^{2}$; radius BMD difference $\left.=0.05 \mathrm{~g} / \mathrm{cm}^{2}\right)$, and in all cases the intermediate (weight/E2) groups had intermediate BMDs. Second, regression models were constructed. Each model included age, weight, E2, and T. These models are shown in Table IV. In models which controlled for the effects of age, weight, and serum $\mathrm{T}$ concentration, serum E2 remained significantly positively associated with BMD at all skeletal sites. Serum T concentrations remained negatively associated with BMD at the spine and trochanter in these same models controlling for E2,
Table III. Correlations: BMD with Sex Steroids

\begin{tabular}{lcccc}
\hline & \multicolumn{3}{c}{ BMD } \\
\cline { 2 - 5 } & Radius & L2-L4 & Femoral neck & Trochanter \\
\hline Height & 0.03 & 0.03 & $0.20^{*}$ & $0.18^{*}$ \\
Weight & 0.15 & $0.25^{\ddagger}$ & $0.35^{\S}$ & $0.39^{\S}$ \\
E1 & 0.11 & 0.09 & 0.12 & 0.15 \\
E2 & $0.28^{\|}$ & $0.21^{\ddagger}$ & $0.33^{\|}$ & $0.35^{\|}$ \\
T & 0.06 & $-0.20^{*}$ & $-0.20^{*}$ & $-0.28^{\|}$ \\
A & -0.07 & $-0.27^{\ddagger}$ & -0.13 & -0.14 \\
DHEA & -0.06 & $-0.20^{*}$ & $-0.20^{*}$ & $-0.25^{\ddagger}$ \\
DHEAS & 0.05 & -0.03 & -0.02 & -0.02 \\
SHBG & -0.07 & -0.17 & -0.08 & $-0.26^{\ddagger}$ \\
& & & & \\
\hline
\end{tabular}

${ }^{\S} P \leq 0.001 ;{ }^{\|} 0.001<P \leq 0.01 ;{ }^{\ddagger} 0.01<P \leq 0.05 ; * 0.05<P<0.10$.

weight, and age. The substitution of other estrogens (E1, $\mathrm{E}_{\mathrm{SO}} \mathrm{S}_{4}$ ) or androgens (A, DHEA, DHEAS) did not improve the models. Similarly, substituting body mass index for weight in the regression models resulted in smaller R-squared values, suggesting a poorer fit. The only significant association with age was positive at the spine, perhaps reflecting an increase in spinal osteoarthritis with age.

To further address the associations of low serum T concentrations with higher BMDs among these men (none of whom had clinical evidence of hypogonadism), we compared the $20 \%$ of the study sample with the lowest serum T concentrations to the $80 \%$ with higher $\mathrm{T}$ concentrations. These men in the bottom $20 \%$ of $\mathrm{T}$ concentrations had mean serum $\mathrm{T}$ of 2.76 $\mathrm{ng} / \mathrm{ml}$, and all were below $3.34 \mathrm{ng} / \mathrm{ml}$ (upper $80 \%$ of the men had serum $\mathrm{T}$ concentrations 3.35-8.68 ng/ml). However, de-

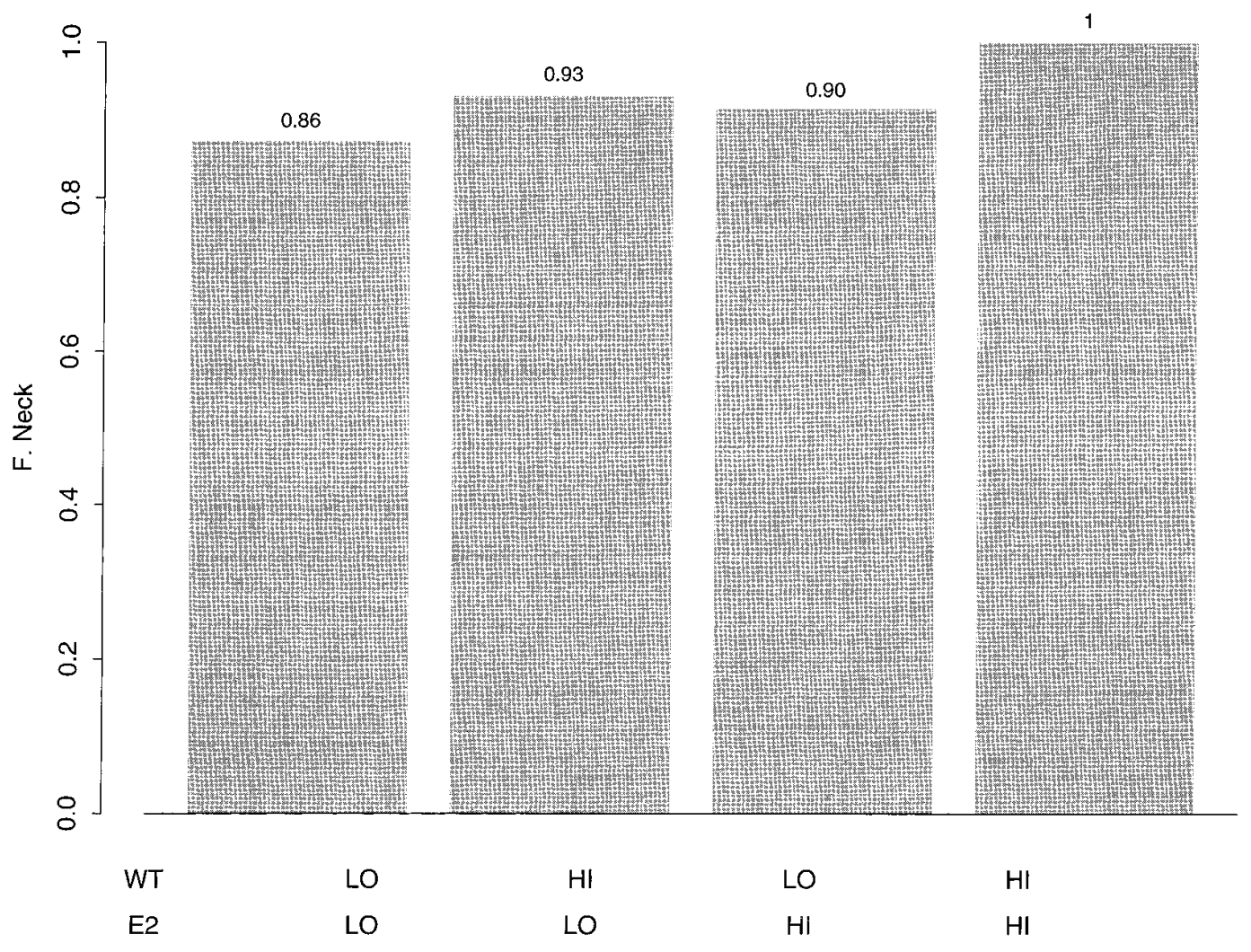

Figure 1. BMD in subjects with serum E2 concentrations below the group median (left pair of bars) and above the median (right pair of bars) stratified by weight. Subjects in the low E2/low weight group (left-most bar) had $0.14 \mathrm{~g} / \mathrm{cm}^{2}$ (1 SD) lower BMDs than those in the upper-most group. Those in the low E2/high weight or high E2/low weight groups had intermediate BMDs. 
Table IV. Multiple Regression Models Predicting BMD

\begin{tabular}{lcccc}
\hline & Radius & L2-L4 & Femoral neck & Trochanter \\
\hline Age, yr & -0.0011 & $0.013^{*}$ & -0.0014 & 0.0017 \\
Weight, $\mathrm{kg}$ & 0.0005 & 0.0028 & $0.0027^{*}$ & $0.0028^{*}$ \\
$\mathrm{E} 2, \mathrm{ln} \mathrm{pg} / \mathrm{ml}$ & $0.087^{*}$ & $0.222^{*}$ & $0.190^{\ddagger}$ & $0.215^{\ddagger}$ \\
$\mathrm{T}, \mathrm{ln} \mathrm{pg} / \mathrm{ml}$ & 0.01 & $-0.19^{*}$ & -0.07 & $-0.14^{*}$ \\
R squared & $10 \%$ & $22 \%$ & $23 \%$ & $28 \%$ \\
& & & & \\
\hline
\end{tabular}

${ }^{\ddagger} P<0.01 ; * P<0.05$.

spite lower total $\mathrm{T}$ concentrations, these men had free $\mathrm{T}$ concentrations that were slightly, but not significantly, higher than the remainder of the sample, due to having $\sim 1$ SD lower SHBG concentrations ( 28.9 vs. $47.2 \mathrm{nM} /$ liter, $P<0.001)$. They were also significantly heavier than men with higher total $\mathrm{T}$ (94.1 vs. $82.8 \mathrm{~kg}, P=0.003$ ).

Finally, to address the issue of bone loss, and to explore whether alterations in rates of skeletal remodeling were associated with serum sex steroids or skeletal characteristics, several exploratory analyses were conducted, although the greater noise/signal ratio in rates of bone loss over this relatively short period somewhat limits these analyses. Those men losing femoral neck bone density at $>1 \%$ per year had significantly higher urinary $\mathrm{Ca} / \mathrm{Cr}$ and $\mathrm{NTX} / \mathrm{Cr}$ ratios, as well as significantly lower serum E1 and $\mathrm{E} \mathrm{SO}_{4}$ concentrations (all $P<$ 0.05). Consistent with the possibility that lower serum estrogens were associated with higher rates of skeletal turnover, the correlations between NTX and serum estrogens were negative (E1, $r=-0.16,0.05<P<0.10$; E2, $r=-0.25, P<0.05$ ). However, despite similar trends at some other skeletal sites, no significant associations with radius or spine bone loss were observed.

\section{Discussion}

Estrogen deficiency associated with menopause is the primary cause of the most rapid phase of bone loss in women (17), and long-term estrogen replacement therapy can prevent half or more of the fractures associated with low bone mass $(20,21)$. No event similar to menopause occurs in men, yet bone loss and fractures associated with low bone mass among men are well documented, and appear to occur at nearly half the rate observed for women. Clinically significant testosterone deficiency can result in low bone mass and osteoporosis, although this is uncommon and accounts for a small fraction of the osteoporosis seen in men (1). Testosterone treatment of acquired hypogonadism in men improves BMD while decreasing bone turnover, much as is seen with estrogen replacement therapy in women, as well as increasing muscle and reducing fat mass (22). The extent to which the skeletal effects of testosterone replacement might be attributed to aromatization of testosterone to estrogen is not known, and therapeutic testosterone may have direct skeletal effects through effects on bone cells (23). However, given that the improvements in BMD in men treated with testosterone are associated with decreased skeletal turnover, rather than increased bone formation, the data suggest that antiresorptive mechanisms, as are seen with estrogen therapy, play the primary role.

Other hormonal causes of bone loss in men have rarely been explored. One small case control study found that men with spinal crush fractures $(n=27)$ had higher bone turnover and lower bone mass, as well as $38 \%$ lower serum E2 concentrations and $11 \%$ higher testosterone concentrations than control subjects $(n=19)$, but these differences in sex steroids were reported as not statistically significant (24). However, they are consistent with what was observed in this study. The positive associations seen here between E2 and BMD are as strong or stronger than those observed between serum estrogens and BMD in women of similar ages (17). Together with previously published data describing specific genetic mutations affecting serum estrogen concentrations or its availability at the tissue level, i.e., those demonstrating severe osteopenia both in a young man with defective estrogen receptors (16) and in another man with aromatase deficiency (25), these findings suggest that estrogens are involved in either the development or the maintenance (or both) of the male skeleton as they are in the female, and that the estrogen/bone mass associations may differ little between the sexes. Given that aromatase activity is the primary determinant of estrogen concentrations in older men and women, further exploration of the role of aromatase activity in the etiology of low bone mass and osteoporotic fractures is perhaps warranted.

The weak negative associations between the androgens and BMD in healthy men were initially surprising, particularly given the reported positive associations between testosterone concentrations and BMD in pre- and perimenopausal women (17). Even those men with the lowest total testosterone concentrations had somewhat higher BMDs than men with the highest testosterone concentrations. However, all of these findings are also consistent with those of the case control study noted above (24). Several elements probably contribute to this observation. First, there are strong inverse associations between testosterone concentrations and body weight (and a positive association between body weight and BMD); however, adjustments for body weight reduced but did not entirely eliminate the negative association. Second, it is also probable that the strong positive association between serum testosterone and SHBG $(r=+0.65)$ in part accounts for the negative relationship between BMD and testosterone. Men with higher testosterone concentrations have higher concentrations of SHBG which bind not only testosterone but also estrogens, yielding similar or lower concentrations of free sex steroids (both estrogens and androgens) than men with lower total testosterone concentrations. Thus, men with higher total testosterone concentrations are thinner and have higher levels of SHBG, with lower concentrations of free sex steroids, and may, as a result of this combination of factors, have somewhat lower BMDs. In postmenopausal women similar processes contribute to lower BMDs. High concentrations of SHBG and low body weight are associated with low bone mass (17). The correlations between SHBG and BMD in women studied in our center ranged between -0.27 (radius) and -0.42 (femoral neck); whereas in men followed over a similar period of time these correlations were between +0.08 and -0.17 . Our analyses of free and non-SHBG bound sex steroids did not, however, yield stronger associations with BMD than those noted for total estrogens or androgens. This may be due in part to the greater difficulty in direct measurements of free sex steroids. The total proportion of the variance in BMD accounted for by sex steroids is also similar in men and women, although in women SHBG showed the strongest associations whereas in men serum E2 was a better predictor of BMD. 
The direct mechanism by which estrogens affect BMD in men is not clear. In women the perimenopausal declines in estrogen are accompanied by increased rates of skeletal remodeling which results in bone loss due to the inability of osteoblasts to fully restore the bone removed by osteoclasts during the resorption phase of skeletal remodeling. Moreover, women with identifiable estrogen deficiencies during growth, e.g., those with anorexia nervosa or activity-induced amenorrhea, have less skeletal mass than do women without these conditions. This study addressed bone loss only in a preliminary manner because of the relatively short period of follow-up of these subjects. There were relatively weak data supporting higher skeletal turnover among men with lower serum estrogen concentrations, and somewhat higher concentrations of turnover markers in those men losing bone more rapidly from the hip. However, longer follow-up will be required to confirm these observations. Men with spinal crush fractures have also been shown to have significantly higher serum concentrations of markers of skeletal remodeling (osteocalcin) (22), and it is possible that this mechanism, which is clearly central to the development of low bone mass in women, is also involved in the development of low bone mass in men. Further study of the role of rates of skeletal remodeling in the etiology of low bone mass in men is clearly indicated.

It is also possible that the estrogen effects observed are related to processes which occurred during the development of peak bone mass, which is also affected by rates of skeletal remodeling (26), or reflect association between estrogen and unmeasured potential confounding influences. Estrogen receptors are present in the bone of both men and women, and it is clear that inadequate exposure to endogenous estrogens in young men can have serious skeletal consequences $(16,25)$. The recent report of osteopenia and continued longitudinal growth in a young adult male with genetically defective estrogen receptors further suggests a role for estrogens in the male skeleton during growth (16). Similarly, the osteopenia and tall stature of a male-female sibling pair with a genetic aromatase deficiency, resulting in very low estrogen concentrations during growth, further support the importance of estrogen in the development of both the male and the female skeletons (25).

Finally, these data and the development of new estrogenlike therapeutic agents raise the possibility of expanding the treatment options for men with low bone mass and osteoporotic fractures. Although high dose estrogen therapy $(2.5$ and $5 \mathrm{mg}$ conjugated equine estrogen per day) for men with previous myocardial infarctions has been attempted, the expected cardiovascular benefits were not observed and the clinical trial was terminated early because of the strong probability that significant estrogen benefits would not be observed should the trial continue on to its originally planned termination (27). However, it is possible that lower doses of estrogen or the use of selective estrogen receptor modulators may result in both skeletal and other benefits for men at high risk of osteoporotic fractures without adverse consequences.

\section{Acknowledgments}

This work was supported by Public Health Service grants P01 AG05793 (Osteoporosis Program Project) and M01 RR00750 (General Clinical Research Center).

\section{References}

1. Orwoll, E.S., and R.F. Klein. 1995. Osteoporosis in men. Endocr. Rev. 16: 87-116.

2. Melton, L.J., W.M. O'Fallon, and B.L. Riggs. 1987. Clinical investigations: secular trends in the incidence of hip fractures. Calcif. Tissue Int. 41:57-64.

3. Kannus, P., J. Parkkari, and S. Niemi. 1995. Age-adjusted incidence of hip fractures. Lancet. 346:50-51.

4. Mann, T., S.K. Oviatt, D. Wilson, D. Nelson, and E.S. Orwoll. 1992. Vertebral deformity in men. J. Bone Miner. Res. 7:1259-1265.

5. Cooper, C., E.J. Atkinson, W.M. O'Fallon, and L.J. Melton. 1992. Incidence of clinically diagnosed vertebral fractures: a population-based study in Rochester, Minnesota, 1985-1989. J. Bone Miner. Res. 7:221-227.

6. Nguyen, T., P. Sambrook, P. Kelly, G. Jones, S. Lord, J. Freund, and J. Eisman. 1993. Prediction of osteoporotic fractures by postural instability and bone density. Bone Miner. 23:1111-1115.

7. Smith, D.M., W.E. Nance, K.W. Kang, J.C. Christian, and C.C. Johnston. 1973. Genetic factors in determining bone mass. J. Clin. Invest. 52:2800-2808.

8. Christian, J.C., P.-L. Yu, C.W. Slemenda, and C.C. Johnston. 1989. Heritability of bone mass: a longitudinal study of aging male twins. Am. J. Hum. Genet. 44:429-433.

9. Snow-Harter, C., R. Whalen, K. Mybrugh, S. Arnaud, and R. Marcus 1992. Bone mineral density, muscle strength, and recreational exercise in men. J. Bone Miner. Res. 7:1291-1296.

10. Slemenda, C.W., J.C. Christian, T. Reed, T.K. Reister, C.J. Williams, and C.C. Johnston, Jr. 1992. Long-term bone loss in men: effects of genetic and environmental factors. Ann. Intern. Med. 117:286-291.

11. Murphy, S., K.-T. Khaw, A. Cassidy, and J.E. Compston. 1992. Sex hormones and bone mineral density in elderly men. Bone Miner. 20:133-140.

12. Drinka, P.J., J. Olson, S. Bauwens, S. Voeks, I. Carlson, and M. Wilson. 1993. Lack of association between free testosterone and bone density in elderly men. Calcif. Tissue Int. 52:67-69.

13. Meier, D.E., E.S. Orwoll, E.J. Keenan, and R.M. Fagerstrom. 1987. Marked decline in trabecular bone mineral content in healthy men with age: lack of association with sex steroid levels. J. Am. Geriatr. Soc. 35:189-197.

14. Anderson, F.H., R.M. Francis, and K. Faulkner. 1996. Androgen supplementation in eugonadal men with osteoporosis: effects of 6 months of treatment on bone mineral density and cardiovascular risk factors. Bone. 18:171-177.

15. Orwoll, E.S., and S. Oviatt. 1992. Transdermal testosterone supplementation in normal older men. J. Bone Miner. Res. 7(Suppl. 1):1617. (Abstr.)

16. Smith, E.P., J. Body, G.R. Frank, H. Takahoashi, R.M. Cohen, B. Specker, and T.C. Williams. 1994. Estrogen resistance caused by a mutation in the estrogen receptor gene in a man. N. Engl. J. Med. 331:1056-1061.

17. Slemenda, C.W., C. Longcope, M.P. Peacock, S.L. Hui, and C.C Johnston. 1996. Sex steroids, bone mass and bone loss. A prospective study of pre-, peri- and postmenopausal women. J. Clin. Invest. 97:14-21.

18. Longcope, C., S.L. Hui, and C.C. Johnston. 1987. Free estradiol, free testosterone and sex hormone binding globulin in peri-menopausal women. $J$. Clin. Endocrinol. Metab. 64:513-518.

19. Eyre, D.R. 1992. New biomarkers of bone resorption. J. Clin. Endocrinol. Metab. 74:470A-470C.

20. Lindsay, R., D.M. Hart, J.M. Aitken, E.B. MacDonald, J.B. Anderson, and A.C. Clarke. 1976. Long-term prevention of postmenopausal osteoporosis by oestrogen: evidence for an increased bone mass after delayed onset of oestrogen treatment. Lancet. 1:1038-1041.

21. Cauley, J.A., D.G. Seeley, K. Ensrud, B. Ettinger, D. Black, and S.R. Cummings. 1995. Estrogen replacement therapy and fractures in older women. Ann. Intern. Med. 122:9-16.

22. Katznelson, L., J.S. Finkelstein, D.A. Schoenfeld, D.I. Rosenthal, E.J Anderson, and A. Klibanski. 1996. Increase in bone density and lean body mass during testosterone administration in men with acquired hypogonadism. J. Clin. Endocrinol. Metab. 81:4358-4365.

23. Kasperk, C.H., J.E. Wergedal, J.R. Farley, T.A. Linkhart, R.T. Turner, and D.J. Baylink. 1989. Androgens directly stimulate proliferation of bone cells in vitro. Endocrinology. 124:1576-1578.

24. Resch, H., P. Pietschmann, W. Woloszczuk, E. Krexner, P. Bernecker, and R. Willvonseder. 1992. Bone mass and biochemical parameters of bone metabolism in men with spinal osteoporosis. Eur. J. Clin. Invest. 22:542-545.

25. Morishima, A., M.M. Grumbach, E.R. Simpson, C. Fisher, and K. Qin. 1995. Aromatase deficiency in male and female siblings caused by a novel mutation and the physiological role of estrogens. J. Clin. Endocrinol. Metab. 80: 3689-3698.

26. Slemenda, C.W., L. Zhou, M. Peacock, S.L. Hui, and C.C. Johnston. 1997. Reduced rates of skeletal remodeling increase peak bone mass. J. Bone Miner. Res. 12:676-682.

27. The Coronary Drug Project Research Group. 1973. The coronary drug project: findings leading to the discontinuation of the $2.5-\mathrm{mg} /$ day estrogen group. J. Am. Med. Assoc. 222:652-657. 